Yong Tae Kim and C. W. Kim, Department of Mathematics, Sungkyunkwan University, Suwon 440-746, Korea. e-mail: wylee@yurim.skku.ac.kr

\title{
OUTER MEASURES GENERATED BY A COUNTABLY ADDITIVE MEASURE ON A RING OF SETS
}

\begin{abstract}
Let $\mathcal{R}$ be any ring of subsets of a set $X$ which is not an algebra and let $\mathcal{A}$ be the algebra generated by $\mathcal{R}$. Suppose that $\mu$ is a countably additive measure on $\mathcal{R}$ and that $\mu^{*}$ is the outer measure generated by $(\mu, \mathcal{R})$. If $X$ is a countable union of sets in $\mathcal{R}$, then there is a unique countably additive measure $\nu$ on $\mathcal{A}$ which extends $\mu$, and the outer measure generated by $(\nu, \mathcal{A})$ coincides with $\mu^{*}$. If $X$ is not a countable union of sets in $\mathcal{R}$, then there exists a family $\left\{\mu_{p}: 0 \leq p \leq \infty\right\}$ of countably additive measures on $\mathcal{A}$ such that each $\mu_{p}$ agrees with $\mu$ on $\mathcal{R}$. For $0 \leq p \leq \infty$, let $\mu_{p}^{*}$ denote the outer measure generated by $\left(\mu_{p}, \mathcal{A}\right)$. Then we have $\mu_{0}^{*} \leq \mu_{p}^{*} \leq \mu_{q}^{*} \leq \mu_{\infty}^{*}=\mu^{*}$ for $0<p<q<\infty$. Moreover, if $\mathcal{M}$ and $\mathcal{M}_{p}$, respectively, denotes the $\sigma$-algebra of $\mu^{*}$-measurable and $\mu_{p}^{*}$-measurable sets, then $\mathcal{M}_{p}=\mathcal{M}_{1} \subset \mathcal{M}_{0}=\mathcal{M}_{\infty}=\mathcal{M}$ for all positive real numbers $p$. As examples, we give countably additive measures on rings for which $\mathcal{M}=\mathcal{M}_{1}$ and $\mathcal{M} \neq \mathcal{M}_{1}$, respectively. By the outer measures generated by $\mu$ we shall mean the outer measures $\mu^{*}$ and $\mu_{p}^{*}$ $(0 \leq p \leq \infty)$.
\end{abstract}

\section{Preliminaries}

Throughout the paper, $X$ denotes a fixed but arbitrary nonempty set unless otherwise stated, and $\mathcal{P}(X)$ denotes the power set of $X$. For each subset $E$ of $X$, let $E^{c}$ denote the complement of $E$ (relative to $X$ ), i.e., $E^{c}=X-E$. By definition, a ring of subsets of $X$ or simply a ring in $\mathrm{X}$ is a nonempty family of subsets of $\mathrm{X}$ which is closed under the formation of unions and differences, and

Key Words: ring, algebra, measure, outer measure, measurability

Mathematical Reviews subject classification: 28A12

Received by the editors June 1, 1999 
an algebra of subsets of $X$ is a ring in $X$ containing $X$. A ring in $X$ which is closed under the formation of countable unions is called a $\sigma$-ring, and a $\sigma$-ring in $\mathrm{X}$ containing $\mathrm{X}$ is called a $\sigma$-algebra. Let $\mathcal{R}$ be any ring in $X$, let $\mathcal{R}^{c}$ be the family of all complements of sets in $\mathcal{R}$, and let $\mathcal{A}$ be the algebra generated by $\mathcal{R}$, that is, the smallest algebra containing $\mathcal{R}$. Trivially $\mathcal{A}=\mathcal{R}=\mathcal{R}^{c}$ if $X \in \mathcal{R}$, and $\mathcal{A}=\mathcal{R} \cup \mathcal{R}^{c}$ if $X \notin \mathcal{R}$. Let $\mathcal{R}_{\sigma}$ and $\mathcal{A}_{\sigma}$ denote the family of all countable unions of sets in $\mathcal{R}$ and $\mathcal{A}$, respectively.

A non-negative extended real-valued set function $\mu$ defined on a ring $\mathcal{R}$ in $X$ is called a measure or a finitely additive measure on $\mathcal{R}$ if $\mu(\emptyset)=0$ and $\mu\left(A_{1} \cup \cdots \cup A_{n}\right)=\mu\left(A_{1}\right)+\cdots+\mu\left(A_{n}\right)$ for every finite collection $\left\{A_{1}, \cdots, A_{n}\right\}$ of pairwise disjoint sets in $\mathcal{R}$. A measure $\mu$ on $\mathcal{R}$ is called a countably additive measure if $\mu\left(\cup_{n=1}^{\infty} A_{n}\right)=\sum_{n=1}^{\infty} \mu\left(A_{n}\right)$ for every pairwise disjoint sequence $\left\{A_{n}\right\}_{n=1}^{\infty}$ of sets in $\mathcal{R}$ whose union is also in $\mathcal{R}$.

Let $\mu$ be a countably additive measure on a ring $\mathcal{R}$ in $X$. For each $E \subset X$, define $\mu^{*}(E)$ by

$$
\mu^{*}(E)=\inf \left\{\sum_{n=1}^{\infty} \mu\left(A_{n}\right): E \subset \cup_{n=1}^{\infty} A_{n}, A_{n} \in \mathcal{R}(n=1,2, \cdots)\right\}
$$

if $E$ can be covered by a set in $\mathcal{R}_{\sigma}$ and otherwise define $\mu^{*}(E)=\infty$ following the convention that $\inf \emptyset=\infty$. Then $\mu^{*}$ is a (Carathéodory) regular outer measure on $X$ which agrees with $\mu$ on $\mathcal{R}$. The outer measure $\mu^{*}$ constructed in this way is called the outer measure generated by $(\mu, \mathcal{R})$ (see, e.g., [2, pp.163165],[3, pp.32-33],[4, pp.36, 41-44]).

For ease of our argument we prove the following simple lemmas.

Lemma 1. Let $\mathcal{R}$ be any ring in $X$ with $X \notin \mathcal{R}$ and $\mathcal{A}=\mathcal{R} \cup \mathcal{R}^{c}$.

(i) If $A \in \mathcal{R}$ and $B \in \mathcal{R}^{c}$, then $A \cup B \in \mathcal{R}^{c}$ and $A \cap B \in \mathcal{R}$.

(ii) If $A, B \in \mathcal{R}^{c}$, then $A \cup B, A \cap B \in \mathcal{R}^{c}$.

(iii) If $A, B \in \mathcal{A}$ and $A \cap B=\emptyset$, then $A \in \mathcal{R}$ or $B \in \mathcal{R}$.

(iv) If $\left\{A_{n}\right\}_{n=1}^{\infty}$ is a pairwise disjoint sequence of sets in $\mathcal{A}$, then there is at most one set $A_{n} \in \mathcal{R}^{c}$.

Proof. Assertions $(i)$ and $(i i)$ follow from de Morgan's laws. To prove (iii), suppose that $A$ and $B$ are disjoint sets in $\mathcal{R}^{c}$. Since $A \subset B^{c} \in \mathcal{R}$, we have $A=A \cap B^{c} \in \mathcal{R}$ by $(i)$. This contradiction proves (iii). Assertion (iv) follows from $($ iii).

Lemma 2. Let $\mathcal{R}$ be any ring in $X$. Then the following assertions are equivalent:

(i) $X \in \mathcal{R}_{\sigma}$;

(ii) $\mathcal{R}^{c} \subset \mathcal{R}_{\sigma} \cap\left(\mathcal{R}_{\sigma}\right)^{c}$;

(iii) $\mathcal{R}_{\sigma} \cap\left(\mathcal{R}_{\sigma}\right)^{c} \neq \emptyset$. 
Proof. Suppose that $X \in \mathcal{R}_{\sigma}$. Assume first that $X \in \mathcal{R}$. Then $\mathcal{R}$ is an algebra so $\mathcal{R}=\mathcal{R}^{c}$. Since $\mathcal{R} \subset \mathcal{R}_{\sigma}$, we have $\mathcal{R}=\mathcal{R}^{c} \subset\left(\mathcal{R}_{\sigma}\right)^{c}$ so that $\mathcal{R}^{c}=\mathcal{R} \subset$ $\mathcal{R}_{\sigma} \cap\left(\mathcal{R}_{\sigma}\right)^{c}$. Next assume that $X \notin \mathcal{R}$. Then $\mathcal{R} \cap \mathcal{R}^{c}=\emptyset$. Let $\left\{X_{n}\right\}_{n=1}^{\infty}$ be any sequence of sets in $\mathcal{R}$ such that $X=\cup_{n=1}^{\infty} X_{n}$. For each $A \in \mathcal{R}^{c}$, we have $A \cap X_{n} \in \mathcal{R}$ for all $n$ by Lemma 1 (i), so $A=\cup_{n=1}^{\infty} A \cap X_{n} \in \mathcal{R}_{\sigma}$, and hence $\mathcal{R}^{c} \subset \mathcal{R}_{\sigma}$. We have $\mathcal{R}^{c} \subset\left(\mathcal{R}_{\sigma}\right)^{c}$, since $\mathcal{R} \subset \mathcal{R}_{\sigma}$. Thus (i) implies (ii). Plainly (ii) implies (iii). Now suppose that (iii) holds, and let $A \in \mathcal{R}_{\sigma} \cap\left(\mathcal{R}_{\sigma}\right)^{c}$. We have $A, A^{c} \in \mathcal{R}_{\sigma}$, so $X=A \cup A^{c} \in \mathcal{R}_{\sigma}$. Thus (iii) implies (i).

The next lemma follows immediately from Lemma 2.

Lemma 3. Let $\mathcal{R}$ be any ring in $X$. Then $X \notin \mathcal{R}_{\sigma}$ if and only if $\mathcal{R}_{\sigma} \cap\left(\mathcal{R}_{\sigma}\right)^{c}=$ $\emptyset$.

Lemma 4. Let $\mathcal{R}$ be any ring in $X$ and let $\mathcal{A}$ be the algebra generated by $\mathcal{R}$.

(i) If $X \in \mathcal{R}_{\sigma}-\mathcal{R}$, then $A_{\sigma}=\mathcal{R}_{\sigma}$.

(ii) If $X \notin \mathcal{R}_{\sigma}$, then, for each $A \in \mathcal{A}_{\sigma}$, one and only one of the following alternatives holds: $A \in \mathcal{R}_{\sigma}$ or $A$ is of the form $E \cup F$, where $E \in \mathcal{R}_{\sigma}$, $F \in \mathcal{R}^{c}$, and $E \cap F=\emptyset$.

Proof. For $(i)$, suppose that $X \in \mathcal{R}_{\sigma}-\mathcal{R}$. Since $X \notin \mathcal{R}$, we have $\mathcal{A}=\mathcal{R} \cup \mathcal{R}^{c}$ so $\mathcal{R}_{\sigma} \subset \mathcal{A}_{\sigma}$. Since $X \in \mathcal{R}_{\sigma}$, we infer from Lemma 2 that $\mathcal{R}^{c} \subset \mathcal{R}_{\sigma}$ so $\mathcal{A} \subset \mathcal{R}_{\sigma}$. Consequently, $\mathcal{A}_{\sigma} \subset \mathcal{R}_{\sigma}$ and hence $\mathcal{A}_{\sigma}=\mathcal{R}_{\sigma}$. Thus $(i)$ is established. To prove (ii), suppose that $X \notin \mathcal{R}_{\sigma}$. Obviously $X \notin \mathcal{R}$, so $\mathcal{A}=\mathcal{R} \cup \mathcal{R}^{c}$. Assume that $A$ is an arbitrary set in $\mathcal{A}_{\sigma}$. Then there is a pairwise disjoint sequence $\left\{A_{n}\right\}_{n=1}^{\infty}$ of sets in $\mathcal{A}$ such that $A=\cup_{n=1}^{\infty} A_{n}$. If $A_{n} \in \mathcal{R}$ for all $n$, then $A \in \mathcal{R}_{\sigma}$. Otherwise, by Lemma 1 ( iv ) there is exactly one set $A_{i} \in \mathcal{R}^{c}$ such that $A_{n} \in \mathcal{R}$ for all $n \neq i$, so that letting $E=\cup_{n \neq i} A_{n}$ and $F=A_{i}$ we have $A=E \cup F$, where $E \in \mathcal{R}_{\sigma}, F \in \mathcal{R}^{c}$, and $E \cap F=\emptyset$. If there were a set $A \in \mathcal{R}_{\sigma}$ of the form $E \cup F$, where $E \in \mathcal{R}_{\sigma}, F \in \mathcal{R}^{c}$, and $E \cap F=\emptyset$, then $F=F \cap A \in \mathcal{R}_{\sigma}$, so $F \in \mathcal{R}_{\sigma} \cap \mathcal{R}^{c}$. This is a contradiction by Lemma 3. Thus (ii) is established.

Assume that $X \notin \mathcal{R}_{\sigma}$ and that $E \in \mathcal{R}_{\sigma}, F \in \mathcal{R}^{c}$, and $E \cap F \neq \emptyset$. We have that $E \cup F \in \mathcal{A}_{\sigma}-\mathcal{R}_{\sigma}$ and $E-F \in \mathcal{R}_{\sigma}$, so $E \cup F=(E-F) \cup F$. Note that $F-E \in\left(\mathcal{R}^{c}\right)_{\delta}=\left(\mathcal{R}_{\sigma}\right)^{c}$ and $F-E$ need not be in $\mathcal{R}^{c}$. Thus we have that every set in $\mathcal{A}_{\sigma}-\mathcal{R}_{\sigma}$ is represented in at least one way as the union of disjoint sets from $\mathcal{R}_{\sigma}$ and $\mathcal{R}^{c}$, respectively, and that such a representation of a set in $\mathcal{A}_{\sigma}-\mathcal{R}_{\sigma}$ need not be unique, for $X=E \cup(X-E)$ for all $E \in \mathcal{R}$.

Example 1. Let $X$ be a nonempty countable set, let $\mathcal{R}$ be the ring of all finite subsets of $X$, and let $\mathcal{A}$ be the algebra generated by $\mathcal{R}$. Trivially 
$\mathcal{R}=\mathcal{A}=\mathcal{P}(X)$ if $X$ is finite. If $X$ is infinite, then $\mathcal{R}_{\sigma}$ is the $\sigma$-ring of all countable subsets of $X$ such that $X \in \mathcal{R}_{\sigma}-\mathcal{R}$, so that $\mathcal{R}_{\sigma}=\mathcal{A}_{\sigma}=\mathcal{P}(X)$.

Example 2. Let $X$ be any uncountable set and let $\mathcal{R}$ be the ring of all finite subsets of $X$. Then $\mathcal{R}_{\sigma}$ is the $\sigma$-ring of all countable subsets of $X$ such that $X \notin \mathcal{R}_{\sigma}$ or, equivalently, $\mathcal{R}_{\sigma} \cap\left(\mathcal{R}_{\sigma}\right)^{c}=\emptyset$ by Lemma 3 . Plainly $\mathcal{R}_{\sigma}$ is the $\sigma$-ring generated by $\mathcal{R}$.

A set $E \subset X$ is called cofinite or cocountable if $E^{c}$ is finite or countable. Let $\mathcal{A}$ and $\mathcal{B}$ denote the algebra and the $\sigma$-algebra generated by $\mathcal{R}$, respectively. Then $\mathcal{A}$ consists of the finite and the cofinite subsets of $X$, i.e., $\mathcal{A}=\mathcal{R} \cup \mathcal{R}^{c}$, and $\mathcal{B}$ consists of the countable and the cocountable subsets of $X$, i.e., $\mathcal{B}=$ $\mathcal{R}_{\sigma} \cup\left(\mathcal{R}_{\sigma}\right)^{c}$. Assert that $\mathcal{A}_{\sigma}=\mathcal{R}_{\sigma} \cup \mathcal{R}^{c}$. Suppose that $A$ is any set in $\mathcal{A}_{\sigma}-\mathcal{R}_{\sigma}$ and let $A=E \cup F$, where $E \in \mathcal{R}_{\sigma}, F \in \mathcal{R}^{c}$ and $E \cap F=\emptyset$. Since $E \subset F^{c} \in \mathcal{R}$, we have $E \in \mathcal{R}$, so $E \cup F \in \mathcal{R}^{c}$ by Lemma 1 (i). Consequently, $\mathcal{A}_{\sigma}-\mathcal{R}_{\sigma}=\mathcal{R}^{c}$ and hence the assertion follows from Lemma 4 (ii). We see readily that $\mathcal{A} \varsubsetneqq \mathcal{B} \varsubsetneqq \mathcal{P}(X)$.

The next lemma is a version of Lemma 3.4 .1 in $[1$, p.76].

Lemma 5. (cf. [5, Problem 9, p.258]) Let $\mathcal{R}$ be an arbitrary ring in $X$ which is not an algebra, let $\mathcal{A}$ be the algebra generated by $\mathcal{R}$, and let $\mu$ be any measure on $\mathcal{R}$.

(i) Define $\mu_{0}$ on $\mathcal{A}$ by $\mu_{0}(E)=\sup \{\mu(A): A \subset E, A \in \mathcal{R}\}$ for all $E$ in $\mathcal{A}$. Then $\mu_{0}$ is a measure on $\mathcal{A}$ such that $\mu_{0}(E)=\mu(E)$ if $E \in \mathcal{R}$.

(ii) For $0<p \leq \infty$, define $\mu_{p}$ on $\mathcal{A}$ by $\mu_{p}(E)=\mu(E)$ if $E \in \mathcal{R}$ and $\mu_{p}(E)=\mu_{0}(E)+p$ if $E \in \mathcal{R}^{c}$. Then $\mu_{p}$ is a measure on $\mathcal{A}$.

(iii) Every measure $\nu$ on $\mathcal{A}$ such that $\nu(E)=\mu(E)$ for all $E$ in $\mathcal{R}$ is of the form $\mu_{p}$ for some $p \in[0, \infty]$.

The measures $\mu_{p}$ are called the measures induced by the measure $\mu$ and parameters $p \in[0, \infty]$.

Proof. We have $\mathcal{A}=\mathcal{R} \cup \mathcal{R}^{c}$. To prove (i), suppose first that $E \in \mathcal{R}$. By the definition of $\mu_{0}$, we have $\mu(E) \leq \mu_{0}(E)$. For any $A \in \mathcal{R}$ with $A \subset E$, we have $\mu(A) \leq \mu(E)$. Taking the supremum of $\mu(A)$ over all such $A$ we obtain $\mu_{0}(E) \leq \mu(E)$ and hence $\mu_{0}(E)=\mu(E)$. To prove the additivity of $\mu_{0}$, suppose that $E$ and $F$ are arbitrary sets in $\mathcal{A}$ such that $E \cap F=\emptyset$. If $E, F \in \mathcal{R}$, then there is nothing to prove. Otherwise, by Lemma 1 (iii) we can assume that $E \in \mathcal{R}$ and $F \in \mathcal{R}^{c}$, so $E \cup F \in \mathcal{R}^{c}$ by Lemma 1 (i). For any $A \in \mathcal{R}$ with $A \subset E \cup F$, we have that $A=(A \cap E) \cup(A \cap F)$, where $A \cap E, A \cap F \in \mathcal{R}$, so

$$
\mu(A)=\mu(A \cap E)+\mu(A \cap F) \leq \mu(E)+\mu_{0}(F)=\mu_{0}(E)+\mu_{0}(F) .
$$


Outer Measures Generated by a Countably Additive Measure239

Taking the supremum of $\mu(A)$ over all $A \in \mathcal{R}$ with $A \subset E \cup F$ we obtain $\mu_{0}(E \cup F) \leq \mu_{0}(E)+\mu_{0}(F)$. To prove the reverse inequality, suppose that $B \in \mathcal{R}$ and $B \subset F$. Since $E \cup F \supset E \cup B \in \mathcal{R}$, we have

$$
\mu(E)+\mu(B)=\mu(E \cup B) \leq \mu_{0}(E \cup F) .
$$

Taking the supremum of $\mu(B)$ over all such $B$ we obtain $\mu_{0}(E)+\mu_{0}(F) \leq$ $\mu_{0}(E \cup F)$, and hence the additivity of $\mu_{0}$ follows. By induction $\mu_{0}$ is finitely additive on $\mathcal{A}$, so (i) is established. To prove (ii), assume that $0<p \leq \infty$ and that $E \in \mathcal{R}, F \in \mathcal{R}^{c}$, and $E \cap F=\emptyset$. Then $E \cup F \in \mathcal{R}^{c}$. By the definition of $\mu_{p}$, together with (i), we have

$$
\mu_{p}(E \cup F)=\mu_{0}(E \cup F)+p=\mu_{0}(E)+\mu_{0}(F)+p=\mu_{p}(E)+\mu_{p}(F) .
$$

By an argument given in the proof of (i) we show that $\mu_{p}$ is additive on $\mathcal{A}$ so it is also finitely additive on $\mathcal{A}$. Thus (ii) is established. To prove (iii), suppose that $\nu$ is any measure on $\mathcal{A}$ which agrees with $\mu$ on $\mathcal{R}$, and let $E$ be an arbitrary set in $\mathcal{R}^{c}$. For any $A \in \mathcal{R}$ with $A \subset E$, we have $\mu(A)=\nu(A) \leq \nu(E)$, so $\mu_{0}(E) \leq \nu(E)$. Therefore, $\mu_{0} \leq \nu$ on $\mathcal{R}^{c}$. If $\nu=\mu_{0}$ on $\mathcal{R}^{c}$, we are done. Suppose that $\mu_{0} \neq \nu$ on $\mathcal{R}^{c}$. Then there is a set $F \in \mathcal{R}^{c}$ such that $\mu_{0}(F)<\nu(F)$, so $0 \leq \mu_{0}(F)<\infty$. Define $p=\nu(F)-\mu_{0}(F)$. We have $0<p \leq \infty$. Assert that $\nu(E)=\mu_{p}(E)$. For this, assume first that $0<p<\infty$ or, equivalently, $0<\nu(F)<\infty$. We have that

$\nu(F)=\nu(E \cap F)+\mu(F-E)<\infty$ and $\mu_{0}(F)=\mu_{0}(E \cap F)+\mu_{0}(F-E)<\infty$.

Since $E-F, F-E \in \mathcal{R}$, we also have that $\nu(E-F)=\mu_{0}(E-F)$ and $\nu(F-E)=\mu_{0}(F-E)$. Then we have $p=\nu(E \cap F)-\mu_{0}(E \cap F)$. Consequently,

$\mu_{p}(E)=\mu_{0}(E)+p=\mu_{0}(E-F)+\mu_{0}(E \cap F)+\nu(E \cap F)-\mu_{0}(E \cap F)=\nu(E)$.

Next assume that $p=\infty$ or, equivalently, $\nu(F)=\infty$. Since $\nu(F)=\nu(E \cap F)+$ $\nu(F-E)$ and $\nu(F-E)=\mu_{0}(F-E) \leq \mu_{0}(F)<\infty$, we obtain $\nu(E \cap F)=\infty$, so $\nu(E)=\infty$. By the definition of $\mu_{\infty}$, we have $\mu_{\infty}(E)=\infty$. Thus the assertion holds and hence (iii) is established.

Lemma 6. Let $\mathcal{R}$ be any ring in $X$ such that $X \notin \mathcal{R}$ and let $\mathcal{A}$ be the algebra generated by $\mathcal{R}$. Suppose that $\mu$ is a countably additive measure on $\mathcal{R}$ and that $\left\{\mu_{p}\right\}$ are the measures on $\mathcal{A}$ that are induced by $\mu$ and parameters $p \in[0, \infty]$. Then:

(i) $\mu_{0}$ is a countably additive measure on $\mathcal{A}$ which extends $\mu$.

(ii) If $X \in \mathcal{R}_{\sigma}$, then $\mu_{0}$ is a unique countably additive measure on 
$\mathcal{A}$ which extends $\mu$.

(iii) If $X \notin \mathcal{R}_{\sigma}$, then each $\mu_{p}$ with $0<p \leq \infty$ is a countably additive measure on $\mathcal{A}$ which extends $\mu$.

(iv) If $X \notin \mathcal{R}_{\sigma}$, then every countably additive measure $\nu$ on $\mathcal{A}$ which extends $\mu$ is of the form $\mu_{p}$ for some $p \in[0, \infty]$.

Proof. For (i), suppose that $\left\{E_{n}\right\}_{n=1}^{\infty}$ is a pairwise disjoint sequence of sets in $\mathcal{A}$ such that $E=\cup_{n=1}^{\infty} E_{n} \in \mathcal{A}$. Assume first that $E \in \mathcal{R}$. By Lemma 1 (i) we have $E_{n} \in \mathcal{R}$ for all $n$, so that by the countable additivity of $\mu$, together with Lemma 5 (i),

$$
\mu_{0}(E)=\mu(E)=\sum_{n=1}^{\infty} \mu\left(E_{n}\right)=\sum_{n=1}^{\infty} \mu_{0}\left(E_{n}\right) .
$$

Next assume that $E \in \mathcal{R}^{c}$ and that $A \in \mathcal{R}$ and $A \subset E$. Since $A=\cup_{n=1}^{\infty} A \cap E_{n}$, where $A \cap E_{n} \in \mathcal{R}$ for all $n$ by Lemma 1 (i), we obtain again by the countable additivity of $\mu$ that $\mu(A)=\sum_{n=1}^{\infty} \mu\left(A \cap E_{n}\right) \leq \sum_{n=1}^{\infty} \mu_{0}\left(E_{n}\right)$. Taking the supremum of $\mu(A)$ over all such $A$ we obtain $\mu_{0}(E) \leq \sum_{n=1}^{\infty} \mu_{0}\left(E_{n}\right)$. Since $\mu_{0}$ is finitely additive on $\mathcal{A}$ by Lemma 5 (i), we have, for any positive integer $n$, $\sum_{i=1}^{n} \mu_{0}\left(E_{i}\right)=\mu_{0}\left(\cup_{i=1}^{n} E_{i}\right) \leq \mu_{0}(E)$. Letting $n \longrightarrow \infty$ we obtain the reverse inequality so that (i) holds.

For (ii), suppose that $X \in \mathcal{R}_{\sigma}$ and that $\nu$ is any countably additive measure on $\mathcal{A}$ which agrees with $\mu$ on $\mathcal{R}$. By part (i), $\nu$ agrees with $\mu_{0}$ on $\mathcal{R}$. Assert that $\nu$ agrees with $\mu_{0}$ on $\mathcal{R}^{c}$. For this, suppose that $A$ is any set in $\mathcal{R}^{c}$. Since $X \in \mathcal{R}_{\sigma}$, we have $\mathcal{R}^{c} \subset \mathcal{R}_{\sigma}$ by Lemma 2 , so that there is a pairwise disjoint sequence $\left\{A_{n}\right\}_{n=1}^{\infty}$ of sets in $\mathcal{R}$ such that $A=\cup_{n=1}^{\infty} A_{n}$. By the countable additivity of $\nu$ and $\mu_{0}$, we obtain $\nu(A)=\sum_{n=1}^{\infty} \nu\left(A_{n}\right)=\sum_{n=1}^{\infty} \mu_{0}\left(A_{n}\right)=$ $\mu_{0}(A)$. Thus the assertion is established and hence (ii) holds.

For (iii), suppose that $X \notin \mathcal{R}_{\sigma}$ and that $0<p \leq \infty$. Trivially $\mu_{p}$ agrees with $\mu$ on $\mathcal{R}$ by the definition of $\mu_{p}$. Let $\left\{E_{n}\right\}_{n=1}^{\infty}$ be any pairwise disjoint sequence of sets in $\mathcal{A}$ such that $E=\cup_{n=1}^{\infty} E_{n} \in \mathcal{A}$. If $E \in \mathcal{R}$, then $E_{n} \in \mathcal{R}$ for all $n$, so

$$
\mu_{p}(E)=\mu(E)=\sum_{n=1}^{\infty} \mu\left(E_{n}\right)=\sum_{n=1}^{\infty} \mu_{p}\left(E_{n}\right),
$$

since $\mu$ is countably additive on $\mathcal{R}$. Suppose that $E \in \mathcal{R}^{c}$. It follows from Lemma 1 (iv), together with Lemma 3, that there is a unique set $E_{i} \in \mathcal{R}^{c}$ such that $E_{n} \in \mathcal{R}$ for all $n \neq i$. Consequently, we obtain from part (i) and the definition of $\mu_{p}$ that

$$
\begin{aligned}
\mu_{p}(E) & =\mu_{0}(E)+p=\sum_{n=1}^{\infty} \mu_{0}\left(E_{n}\right)+p=\mu_{0}\left(E_{i}\right)+p+\sum_{n \neq i} \mu\left(E_{n}\right) \\
& =\mu_{p}\left(E_{i}\right)+\sum_{n \neq i} \mu_{p}\left(E_{n}\right)=\sum_{n=1}^{\infty} \mu_{p}\left(E_{n}\right) .
\end{aligned}
$$


Outer Measures Generated By a Countably Additive Measure241

Thus (iii) holds. Assertion (iv) follows from Lemma 5 (iii), together with parts (i) and (iii).

Suppose that $\mathcal{R}$ is a $\sigma$-ring in $X$ which is not a $\sigma$-algebra and that $\mathcal{A}$ is the $\sigma$-algebra generated by $\mathcal{R}$. Let $\mu$ be any countably additive measure on $\mathcal{R}$. By parts (i), (iii) and (iv) of Lemma 6, the measures $\mu_{p} \quad(0 \leq p \leq \infty)$ are the only countably additive measures on $\mathcal{A}$ which extend $\mu$ (see $[5$, Problem 9, p.258]).

Let $\mathcal{R}$ be any ring in $X$. It is easy to give an alternate, but equivalent, definition of $\mathcal{R}_{\sigma}$ : define $\mathcal{R}_{\sigma}$ as the family of unions of increasing sequences of sets in $\mathcal{R}$. Notice that $\mathcal{R}_{\sigma}$ is the smallest family of subsets of $X$ containing $\mathcal{R}$ and closed under the formation of finite intersections and countable unions. Let $\mu$ be a countably additive measure on $\mathcal{R}$. Define $\bar{\mu}$ on $\mathcal{R}_{\sigma}$ as follows : for each $A \in \mathcal{R}_{\sigma}$, let $\bar{\mu}(A)=\lim _{n} \mu\left(A_{n}\right)$, where $\left\{A_{n}\right\}_{n=1}^{\infty}$ is any increasing sequence of sets in $\mathcal{R}$ such that $A=\cup_{n=1}^{\infty} A_{n}$. We show readily that $\bar{\mu}$ is defined unambiguously on $\mathcal{R}_{\sigma}$. Furthermore, $\bar{\mu}$ is a unique monotone increasing, countably additive non-negative extended real-valued set function on $\mathcal{R}_{\sigma}$ which agrees with $\mu$ on $\mathcal{R}$. We also have that $\bar{\mu}(A)=\sup \{\mu(B): B \subset A, \quad B \in \mathcal{R}\}$ for all $A$ in $\mathcal{R}_{\sigma}$. For the outer measure $\mu^{*}$ generated by $(\mu, \mathcal{R})$, we obtain that $\mu^{*}(E)=\inf \left\{\bar{\mu}(A): E \subset A \in \mathcal{R}_{\sigma}\right\}$ if $E$ can be covered by a set in $\mathcal{R}_{\sigma}$ and $\mu^{*}(E)=\infty$ otherwise.

Lemma 7. Let $\mathcal{R}$ be any ring in $X$ such that $X \in \mathcal{R}_{\sigma}-\mathcal{R}$ and let $\mathcal{A}$ be the algebra generated by $\mathcal{R}$. Suppose that $\mu$ is a countably additive measure on $\mathcal{R}$ and that $\mu_{0}$ is the unique countably additive measure on $\mathcal{A}$ extending $\mu$. Then $\mathcal{R}_{\sigma}=\mathcal{A}_{\sigma}$ and $\bar{\mu}=\overline{\left(\mu_{0}\right)}$ on $\mathcal{R}_{\sigma}$.

Proof. The first equality follows from Lemma 4 (i). The set functions $\bar{\mu}$ and $\overline{\left(\mu_{0}\right)}$ denotes, respectively, the extension of $\mu$ to $\mathcal{R}_{\sigma}$ and $\mu_{0}$ to $\mathcal{A}_{\sigma}$. Thus both $\bar{\mu}$ and $\overline{\left(\mu_{0}\right)}$ are defined on $\mathcal{R}_{\sigma}=A_{\sigma}$. Suppose that $A$ is an arbitrary set in $\mathcal{R}_{\sigma}$ and that $\left\{A_{n}\right\}_{n=1}^{\infty}$ is an increasing sequence of sets in $\mathcal{R}$ such that $A=$ $\cup_{n=1}^{\infty} A_{n}$. By the definitions of $\bar{\mu}$ and $\overline{\left(\mu_{0}\right)}$ we obtain that $\bar{\mu}(A)=\lim _{n} \mu\left(A_{n}\right)=$ $\lim _{n} \mu_{0}\left(A_{n}\right)=\overline{\left(\mu_{0}\right)}(A)$. Thus the lemma is established.

Theorem 1. Let $X, \mathcal{R}, \mathcal{A}, \mu$ and $\mu_{0}$ be as in Lemma 7. Then $\mu^{*}(E)=\mu_{0}^{*}(E)$ for all $E \subset X$.

Proof. Suppose that $E$ is an arbitrary subset of $X$. Then $E$ has a cover in $\mathcal{R}_{\sigma}$, since $E \subset X \in \mathcal{A}_{\sigma}=\mathcal{R}_{\sigma}$ by the first part of Lemma 7 . We obtain from the second part of Lemma 7 that

$\mu^{*}(E)=\inf \left\{\bar{\mu}(A): E \subset A \in \mathcal{R}_{\sigma}\right\}=\inf \left\{\overline{\left(\mu_{0}\right)}(A): E \subset A \in \mathcal{A}_{\sigma}\right\}=\mu_{0}^{*}(E)$. 
Thus the theorem is established.

Example 3. Let $X$ denote the real line $\mathbb{R}$ and let $\mathcal{R}$ denote the ring of all unions of finite collections of pairwise disjoint intervals $[a, b)$, where $-\infty<a \leq$ $b<\infty$. Obviously $X \in \mathcal{R}_{\sigma}-\mathcal{R}$. For any finite collection $\left\{\left[a_{i}, b_{i}\right), i=1, \cdots, n\right\}$ of pairwise disjoint intervals in $\mathcal{R}$, define $\mu\left(\cup_{i=1}^{n}\left[a_{i}, b_{i}\right)\right)=\sum_{i=1}^{n}\left(b_{i}-a_{i}\right)$. Then $\mu$ is a countably additive measure on $\mathcal{R}$. Let $\mathcal{A}=\mathcal{R} \cup \mathcal{R}^{c}$ and let $\mu_{0}$ denote the unique countably additive measure on $\mathcal{A}$ which extends $\mu$. We have $\mu_{0}(E)=\infty$ for all $E \in \mathcal{R}^{c}$. By Theorem 1 we have $\mu^{*}=\mu_{0}^{*}$. The outer measure $\mu^{*}$ is called Lebesgue outer measure on $\mathbb{R}$.

For any subset $E$ of $X$, card $E$ denotes the cardinal number of $E$.

Example 4. Let $X$ denote the set $\mathbb{N}$ of all positive integers and let $\mathcal{R}$ denote the ring of all finite subsets of $X$. Then $X \notin \mathcal{R}$. The algebra $\mathcal{A}$ generated by $\mathcal{R}$ consists of the finite and the cofinite subsets of $X$ so $\mathcal{A} \varsubsetneqq \mathcal{R}_{\sigma}=\mathcal{A}_{\sigma}=\mathcal{P}(X)$. Suppose that $\mu$ is any measure on $\mathcal{R}$. Trivially $\mu$ is countably additive on $\mathcal{R}$. Let $\mu_{0}$ and $\bar{\mu}$ denote the countably additive measure extending $\mu$ to $\mathcal{A}$ and $\mathcal{P}(X)$, respectively. We have that $\mu_{0}(E)=\sum_{n \in E} \mu(\{n\})$ for all $E \in \mathcal{A}$ and $\bar{\mu}(E)=\sum_{n \in E} \mu(\{n\})$ for all $E \subset X$. Then $\bar{\mu}$ is a countably additive measure on $\mathcal{P}(X)$ such that $\bar{\mu}(E)=\mu^{*}(E)=\mu_{0}^{*}(E)$ for all $E \in \mathcal{P}(X)$.

Next suppose that $\mu(E)=\operatorname{card} E$ for all $E \in \mathcal{R}$. Plainly $\mu$ is a measure on $\mathcal{R}$. Then the countably additive measure $\bar{\mu}$ on $\mathcal{P}(X)$ is counting measure, that is, $\bar{\mu}(E)=n$ if $E$ is finite and has $n$ elements and $\bar{\mu}(E)=\infty$ if $E$ is infinite.

\section{$2 \quad$ Main Result and Examples}

Throughout this section we shall assume that $\mathcal{R}$ is an arbitrary ring in $X$ such that $X \notin \mathcal{R}_{\sigma}, \mathcal{A}$ is the algebra generated by $\mathcal{R}$, and $\mu$ is any countably additive measure on $\mathcal{R}$. Let $\bar{\mu}$ denote the unique extension of $\mu$ from $\mathcal{R}$ to $\mathcal{R}_{\sigma}$ and $\mu^{*}$ the outer measure generated by $(\mu, \mathcal{R})$. Form the countably additive measures $\mu_{p}(0 \leq p \leq \infty)$ on $\mathcal{A}$ that are induced by $\mu$ and parameters $p \in[0, \infty]$. For $0 \leq p \leq \infty$, let $\overline{\left(\mu_{p}\right)}$ denote the unique extension of $\mu_{p}$ from $\mathcal{A}$ to $\mathcal{A}_{\sigma}$, and let $\mu_{p}^{*}$ denote the outer measure generated by $\left(\mu_{p}, \mathcal{A}\right)$. Let $\mathcal{M}$ and $\mathcal{M}_{p}(0 \leq p \leq \infty)$ denote the $\sigma$-algebra of all $\mu^{*}$-measurable and $\mu_{p}^{*}$-measurable subsets of $X$, respectively.

Lemma 8. Define $(\bar{\mu})_{0}$ on $\mathcal{A}_{\sigma}$ by $(\bar{\mu})_{0}(E)=\bar{\mu}(E)$ if $E \in \mathcal{R}_{\sigma}$ and $(\bar{\mu})_{0}(E \cup$ $F)=\sup \left\{\bar{\mu}(A): A \subset E \cup F, A \in \mathcal{R}_{\sigma}\right\}$ if $E \in \mathcal{R}_{\sigma}, F \in \mathcal{R}^{c}$, and $E \cap F=\emptyset$. Then:

(i) $(\bar{\mu})_{0}(E)=\overline{\left(\mu_{0}\right)}(E)=\bar{\mu}(E)$ if $E \in \mathcal{R}_{\sigma}$;

(ii) $(\bar{\mu})_{0}(F)=\overline{\left(\mu_{0}\right)}(F)=\mu_{0}(F)$ if $F \in \mathcal{R}^{c}$; 
Outer Measures Generated by a Countably Additive Measure243

(iii) $(\bar{\mu})_{0}(E \cup F)=\bar{\mu}(E)+\mu_{0}(F)$ if $E \in \mathcal{R}_{\sigma}, F \in \mathcal{R}^{c}$, and $E \cap F=\emptyset$;

(iv) $(\bar{\mu})_{0}$ is defined unambiguously on $\mathcal{A}_{\sigma}-\mathcal{R}_{\sigma}$;

(v) $(\bar{\mu})_{0}(A)=\overline{\left(\mu_{0}\right)}(A)$ for all $A \in \mathcal{A}_{\sigma}$.

Proof. For (i), suppose that $E \in \mathcal{R}_{\sigma}$ and that $\left\{E_{n}\right\}_{n=1}^{\infty}$ is an increasing sequence of sets in $\mathcal{R}$ such that $E=\cup_{n=1}^{\infty} E_{n}$. We have that $(\bar{\mu})_{0}(E)=$ $\bar{\mu}(E)=\lim _{n} \mu\left(E_{n}\right)=\lim _{n} \mu_{0}\left(E_{n}\right)=\overline{\left(\mu_{0}\right)}(E)$, so that (i) holds.

For (ii), suppose that $F \in \mathcal{R}^{c}$. By the definitions of $(\bar{\mu})_{0}$ and $\overline{\left(\mu_{0}\right)}$, we have that $(\bar{\mu})_{0}(F) \geq \mu_{0}(F)=\overline{\left(\mu_{0}\right)}(F)$. To prove the reverse inequality, suppose that $A$ is any set in $\mathcal{R}_{\sigma}$ such that $A \subset F$, and let $\left\{A_{n}\right\}_{n=1}^{\infty}$ be an increasing sequence of sets in $\mathcal{R}$ such that $A=\cup_{n=1}^{\infty} A_{n}$. Since $\mu\left(A_{n}\right) \leq \mu_{0}(F)$ for all $n$, we obtain that $\bar{\mu}(A)=\lim _{n} \mu\left(A_{n}\right) \leq \mu_{0}(F)$. Taking the supremum of $\bar{\mu}(A)$ over all $A \in \mathcal{R}_{\sigma}$ with $A \subset F$ we get $(\bar{\mu})_{0}(F) \leq \mu_{0}(F)$. Thus (ii) holds.

For (iii), suppose that $E \in \mathcal{R}_{\sigma}, F \in \mathcal{R}^{c}$, and $E \cap F=\emptyset$, and let $A$ be any set in $\mathcal{R}_{\sigma}$ such that $A \subset E \cup F$. Then $A=(A \cap E) \cup(A \cap F)$, where $A \cap E, A \cap F \in \mathcal{R}_{\sigma}$ and $A \cap E \cap F=\emptyset$. Since $\bar{\mu}$ is monotone increasing and countably additive on $\mathcal{R}_{\sigma}$, we obtain from (ii) that

$$
\bar{\mu}(A)=\bar{\mu}(A \cap E)+\bar{\mu}(A \cap F) \leq \bar{\mu}(E)+(\bar{\mu})_{0}(F)=\bar{\mu}(E)+\mu_{0}(F) .
$$

Taking the supremum of $\bar{\mu}(A)$ over all such $A$ we have that $(\bar{\mu})_{0}(E \cup F) \leq$ $\bar{\mu}(E)+\mu_{0}(F)$. To prove the reverse inequality, suppose that $B \subset F$ and $B \in \mathcal{R}$. Since $E \cup B \in \mathcal{R}_{\sigma}$ and $E \cup B \subset E \cup F$, we have that

$$
\bar{\mu}(E)+\mu(B)=\bar{\mu}(E)+\bar{\mu}(B)=\bar{\mu}(E \cup B) \leq(\bar{\mu})_{0}(E \cup F) .
$$

Taking the supremum of $\mu(B)$ over all $B \in \mathcal{R}$ with $B \subset F$ we have that $\bar{\mu}(E)+\mu_{0}(F) \leq(\bar{\mu})_{0}(E \cup F)$. Thus (iii) holds.

For (iv), suppose that $A$ is any set in $\mathcal{A}_{\sigma}-\mathcal{R}_{\sigma}$ and that $A=E_{1} \cup F_{1}=$ $E_{2} \cup F_{2}$, where $E_{i} \in \mathcal{R}_{\sigma}, F_{i} \in \mathcal{R}^{c}$, and $E_{i} \cap F_{i}=\emptyset$ for $i=1$, 2. Since $E_{1}$ is the union of two disjoint sets $E_{1} \cap E_{2}$ and $E_{1} \cap F_{2}$ that are in $\mathcal{R}_{\sigma}$, we obtain that $\bar{\mu}\left(E_{1}\right)=\bar{\mu}\left(E_{1} \cap E_{2}\right)+\bar{\mu}\left(E_{1} \cap F_{2}\right)$. Since $F_{1}=\left(F_{1} \cap E_{2}\right) \cup\left(F_{1} \cap F_{2}\right)$, where $F_{1} \cap E_{2} \in \mathcal{R}_{\sigma}, F_{1} \cap F_{2} \in \mathcal{R}^{c}$ and $F_{1} \cap E_{2} \cap F_{2}=\emptyset$, we have from (ii) and (iii) that $\mu_{0}\left(F_{1}\right)=(\bar{\mu})_{0}\left(F_{1}\right)=\bar{\mu}\left(F_{1} \cap E_{2}\right)+\mu_{0}\left(F_{1} \cap F_{2}\right)$. Consequently, again by (iii) we obtain that

$$
\begin{aligned}
(\bar{\mu})_{0}\left(E_{1} \cup F_{1}\right) & =\bar{\mu}\left(E_{1}\right)+\mu_{0}\left(F_{1}\right) \\
& =\bar{\mu}\left(E_{1} \cap E_{2}\right)+\bar{\mu}\left(E_{1} \cap F_{2}\right)+\bar{\mu}\left(F_{1} \cap E_{2}\right)+\mu_{0}\left(F_{1} \cap F_{2}\right) .
\end{aligned}
$$

Interchanging $E_{1}$ and $F_{1}$ by $E_{2}$ and $F_{2}$, respectively, in the above equations we see at once that $(\bar{\mu})_{0}\left(E_{1} \cup F_{1}\right)=(\bar{\mu})_{0}\left(E_{2} \cup F_{2}\right)$. Thus (iv) holds.

For (v), suppose that $A$ is any set in $\mathcal{A}_{\sigma}-\mathcal{R}_{\sigma}$ of the form $E \cup F$, where 
$E \in \mathcal{R}_{\sigma}, F \in \mathcal{R}^{c}$, and $E \cap F=\emptyset$. Let $\left\{E_{n}\right\}_{n=1}^{\infty}$ be an increasing sequence of sets in $\mathcal{R}$ with $E=\cup_{n=1}^{\infty} E_{n}$. Since $\left\{E_{n} \cup F\right\}_{n=1}^{\infty}$ is an increasing sequence of sets in $\mathcal{A}$ with $A=\cup_{n=1}^{\infty}\left(E_{n} \cup F\right)$, we obtain that

$$
\begin{aligned}
\overline{\left(\mu_{0}\right)}(A) & =\lim _{n} \mu_{0}\left(E_{n} \cup F\right) \\
& =\lim _{n} \mu_{0}\left(E_{n}\right)+\mu_{0}(F)=\lim _{n} \mu\left(E_{n}\right)+\mu_{0}(F)=\bar{\mu}(E)+\mu_{0}(F),
\end{aligned}
$$

so by (iii), $\overline{\left(\mu_{0}\right)}(A)=(\bar{\mu})_{0}(A)$. Now Assertion (v) follows from (i) and Lemma 4 (ii).

The simple proof of the next lemma is omitted.

Lemma 9. For $0<p \leq \infty$, define $(\bar{\mu})_{p}$ on $\mathcal{A}_{\sigma}$ by $(\bar{\mu})_{p}(A)=\bar{\mu}(A)$ if $A \in \mathcal{R}_{\sigma}$ and $(\bar{\mu})_{p}(A)=(\bar{\mu})_{0}(A)+p$ if $A \in \mathcal{A}_{\sigma}-\mathcal{R}_{\sigma}$. Then :

(i) $(\bar{\mu})_{p}(E)=\overline{\left(\mu_{p}\right)}(E)=\bar{\mu}(E)$ if $E \in \mathcal{R}_{\sigma}$;

(ii) $(\bar{\mu})_{p}(F)=\overline{\left(\mu_{p}\right)}(F)=\mu_{p}(F)$ if $F \in \mathcal{R}^{c}$;

and $E \cap F=\emptyset$;

(iii) $(\bar{\mu})_{p}(E \cup F)=\overline{\left(\mu_{p}\right)}(E \cup F)=\bar{\mu}(E)+\mu_{p}(F)$ if $E \in \mathcal{R}_{\sigma}, F \in \mathcal{R}^{c}$,

(iv) $(\bar{\mu})_{p}(A)=\overline{\left(\mu_{p}\right)}(A)$ for all $A \in \mathcal{A}_{\sigma}$.

For $0 \leq p \leq \infty$, we write $\bar{\mu}_{p}(A)$ for $(\bar{\mu})_{p}(A)=\overline{\left(\mu_{p}\right)}(A)$, where $A \in \mathcal{A}_{\sigma}$.

Lemma 10. Let $E$ be any subset of $X$.

(i) If $E$ has a cover in $\mathcal{R}_{\sigma}$, then $\mu_{p}^{*}(E)=\mu^{*}(E)$ for all $p \in[0, \infty]$.

(ii) If $E$ has no cover in $\mathcal{R}_{\sigma}$, then $\mu_{p}^{*}(E)=\inf \left\{\bar{\mu}_{p}(A): E \subset A \in \mathcal{A}_{\sigma}-\mathcal{R}_{\sigma}\right\}$ for all $p \in[0, \infty]$.

(iii) If $E$ has no cover in $\mathcal{R}_{\sigma}$, then $\mu_{p}^{*}(E)=\mu_{0}^{*}(E)+p$ for all $p \in(0, \infty)$, and $\mu_{\infty}^{*}(E)=\infty$.

$\infty$

(iv) $\mu_{0}^{*}(E) \leq \mu_{p}^{*}(E) \leq \mu_{q}^{*}(E) \leq \mu_{\infty}^{*}(E)=\mu^{*}(E)$, where $0<p<q<$

Proof. To prove (i), suppose that $E \subset C \in \mathcal{R}_{\sigma}$ and that $p \in[0, \infty]$. Let $\mathcal{C}$ and $\mathcal{D}$ denote the family of all coverings of $E$ from $\mathcal{R}_{\sigma}$ and $\mathcal{A}_{\sigma}$, respectively. We have that $C \in \mathcal{C} \subset \mathcal{D}$ so by part (i) of Lemma 8 or 9 ,

$$
\begin{aligned}
\mu_{p}^{*}(E) & =\inf \left\{\bar{\mu}_{p}(D): D \in \mathcal{D}\right\} \leq \inf \left\{\bar{\mu}_{p}(D): D \in \mathcal{C}\right\} \\
& =\inf \{\bar{\mu}(D): D \in \mathcal{C}\}=\mu^{*}(E) .
\end{aligned}
$$

Trivially the reverse inequality holds if $\mu_{p}^{*}(E)=\infty$. To complete the proof of (i), assume first that $0 \leq p<\infty$ and $\mu_{p}^{*}(E)<\infty$. For any $\epsilon>0$, there 
is an $A \in \mathcal{A}_{\sigma}$ such that $E \subset A$ and $\bar{\mu}_{p}(A) \leq \mu_{p}^{*}(E)+\epsilon$. Since $E \subset A \cap$ $C \in \mathcal{R}_{\sigma}$, we have from part (i) of Lemma 8 or 9 that $\mu^{*}(E) \leq \bar{\mu}(A \cap C)=$ $\bar{\mu}_{p}(A \cap C) \leq \overline{\mu_{p}}(A) \leq \mu_{p}^{*}(E)+\epsilon$, and hence $\mu^{*}(E) \leq \mu_{p}^{*}(E)$, since $\epsilon$ is an arbitrary positive real number. Next assume that $\mu_{\infty}^{*}(E)<\infty$. Then we have $\mu_{\infty}^{*}(E)=\inf \left\{\bar{\mu}_{\infty}(A): E \subset A \in \mathcal{A}_{\sigma}\right\}<\infty$. By the definition of $\mu_{\infty}$ we have that $\mu_{\infty}(F)=\infty$ for all $F \in \mathcal{R}^{c}$, so that $\bar{\mu}_{\infty}(A)=\infty$ for all $A \in \mathcal{A}_{\sigma}-\mathcal{R}_{\sigma}$ by Lemma 9 (iii). Consequently,

$\mu_{\infty}^{*}(E)=\inf \left\{\bar{\mu}_{\infty}(A): E \subset A \in \mathcal{R}_{\sigma}\right\}=\inf \left\{\bar{\mu}(A): E \subset A \in \mathcal{R}_{\sigma}\right\}=\mu^{*}(E)$

by Lemma 4 (ii), together with Lemma 9 (i). Thus (i) is established.

To prove (ii), suppose that $E$ has no cover in $\mathcal{R}_{\sigma}$. Since $E \subset X \in \mathcal{R}^{c} \subset$ $\mathcal{A}_{\sigma}-\mathcal{R}_{\sigma}, E$ has at least one cover from $\mathcal{A}_{\sigma}-\mathcal{R}_{\sigma}$ so by Lemma 4 (ii) we establish (ii).

To prove (iii), again suppose that $E$ has no cover in $\mathcal{R}_{\sigma}$. Assume first that $0<p<\infty$. Since $\bar{\mu}_{p}(A)=\bar{\mu}_{0}(A)+p$ for all $A \in \mathcal{A}_{\sigma}-\mathcal{R}_{\sigma}$ by Lemma 9 , we obtain from (ii) that $\mu_{p}^{*}(E)=\inf \left\{\bar{\mu}_{0}(A)+p: E \subset A \in \mathcal{A}_{\sigma}-\mathcal{R}_{\sigma}\right\}=\mu_{0}^{*}(E)+p$. As we noted in the proof of (i), we have that $\bar{\mu}_{\infty}(A)=\infty$ for all $A \in \mathcal{A}_{\sigma}-\mathcal{R}_{\sigma}$, so that by (ii), we get $\mu_{\infty}^{*}(E)=\infty$. Thus (iii) is established.

If $E$ has no cover in $\mathcal{R}_{\sigma}$, then by the definition of $\mu^{*}$, together with (iii), we have $\mu^{*}(E)=\mu_{\infty}^{*}(E)=\infty$. By (i), we have $\mu^{*}(E)=\mu_{\infty}^{*}(E)$. The inequalities in (iv) now follow from (i) and (iii). Thus (iv) is established.

We now turn to the relations among the $\sigma$-algebras $\mathcal{M}$ and $\mathcal{M}_{p}$ with $0 \leq$ $p \leq \infty$. Since $\mu^{*}=\mu_{\infty}^{*}$ by Lemma 10 (iv), we obtain $\mathcal{M}=\mathcal{M}_{\infty}$. The next proposition shows that $\mathcal{M}_{p} \subset \mathcal{M}$ for all $p \in[0, \infty)$.

Proposition 1. For $0 \leq p<\infty$, every $\mu_{p}^{*}$-measurable subset of $X$ is $\mu^{*}$ measurable.

Proof. Suppose that $E$ is an arbitrary $\mu_{p}^{*}$-measurable subset of $X$, and let $T$ be any subset of $X$ with $\mu^{*}(T)<\infty$. Obviously $T$ has a cover in $\mathcal{R}_{\sigma}$, so the same is true for $T \cap E$ and $T \cap E^{c}$, respectively. By Lemma 10 (i) we have that $\mu_{p}^{*}(T)=\mu^{*}(T), \mu_{p}^{*}(T \cap E)=\mu^{*}(T \cap E)$, and $\mu_{p}^{*}\left(T \cap E^{c}\right)=\mu^{*}\left(T \cap E^{c}\right)$. Consequently, by the $\mu_{p}^{*}$-measurability of $E$ we obtain that

$$
\mu^{*}(T)=\mu_{p}^{*}(T)=\mu_{p}^{*}(T \cap E)+\mu_{p}^{*}\left(T \cap E^{c}\right)=\mu^{*}(T \cap E)+\mu^{*}\left(T \cap E^{c}\right),
$$

and hence $E$ is $\mu^{*}$-measurable.

Proposition 2. For each $E \subset X, E$ is $\mu^{*}$-measurable if and only if $E$ is $\mu_{0}^{*}$-measurable. 
Proof. Suppose that $E$ is $\mu^{*}$-measurable and that $T$ is any subset of $X$ with $\mu_{0}^{*}(T)<\infty$. First assume that $T$ has a cover in $\mathcal{R}_{\sigma}$. Then $T \cap E$ and $T \cap E^{c}$, respectively, has a cover in $\mathcal{R}_{\sigma}$. Since $E$ is $\mu^{*}$-measurable, using Lemma 10 (i) we obtain that

$$
\mu_{0}^{*}(T)=\mu^{*}(T)=\mu^{*}(T \cap E)+\mu^{*}\left(T \cap E^{c}\right)=\mu_{0}^{*}(T \cap E)+\mu_{0}^{*}\left(T \cap E^{c}\right),
$$

and hence $E$ is $\mu_{0}^{*}$-measurable.

Next assume that $T$ has no cover in $\mathcal{R}_{\sigma}$. By Lemma 10 (ii) there is, for any $\epsilon>0$, a set $A=F \cup G$, where $F \in \mathcal{R}_{\sigma}, G \in \mathcal{R}^{c}$ and $F \cap G=\emptyset$, such that $T \subset A$ and $\bar{\mu}_{0}(A) \leq \mu_{0}^{*}(T)+\epsilon$. By Lemma 8 we have that $\bar{\mu}_{0}(A)=\bar{\mu}(F)+\mu_{0}(G)$. We show easily that there is a set $H \in \mathcal{R}_{\sigma}$ such that $H \subset G$ and $\mu_{0}(G)=$ $\bar{\mu}(H)$. Set $B=F \cup H$. We have that $B \in \mathcal{R}_{\sigma}, B \subset A$ and $\bar{\mu}_{0}(A)=\bar{\mu}(B)$. Since $A-B=G-H$ is $\mu_{0}^{*}$-measurable and since $\mu_{0}(G)<\infty$, we have that $\mu_{0}^{*}(A-B)=\mu_{0}^{*}(G-H)=\mu_{0}(G)-\bar{\mu}(H)=0$. Since, for any $C \subset X, \mu_{0}^{*}$ is countably additive on the trace of $\mathcal{M}_{0}$ on $C$, i.e., $\left\{S \cap C: S \in \mathcal{M}_{0}\right\}$ (see, e.g., [5, Problem 2, p.291]), we have that $\mu_{0}^{*}(A \cap C)=\mu_{0}^{*}(B \cap C)$. In particular, we have that $\mu_{0}^{*}(A \cap E)=\mu_{0}^{*}(B \cap E)$ and $\mu_{0}^{*}\left(A \cap E^{c}\right)=\mu_{0}^{*}\left(B \cap E^{c}\right)$.

Consequently, using the $\mu^{*}$-measurability of $B$ and $E$, together with Lemma 10 (i), we obtain that

$$
\begin{aligned}
\mu_{0}^{*}(T)+\epsilon \geq \bar{\mu}_{0}(A) & =\bar{\mu}(B)=\mu^{*}(B \cap E)+\mu^{*}\left(B \cap E^{c}\right) \\
& =\mu_{0}^{*}(B \cap E)+\mu_{0}^{*}\left(B \cap E^{c}\right) \\
& =\mu_{0}^{*}(A \cap E)+\mu_{0}^{*}\left(A \cap E^{c}\right) \geq \mu_{0}^{*}(T \cap E)+\mu_{0}^{*}\left(T \cap E^{c}\right),
\end{aligned}
$$

and hence $\mu_{0}^{*}(T) \geq \mu_{0}^{*}(T \cap E)+\mu_{0}^{*}\left(T \cap E^{c}\right)$, since $\epsilon$ is an arbitrary positive real number. Thus $E$ is $\mu_{0}^{*}$-measurable. By Proposition 1 we establish the proposition.

Proposition 3. For any positive real numbers $p$ and $q$, a subset $E$ of $X$ is $\mu_{p}^{*}$-measurable if and only if $E$ is $\mu_{q}^{*}$-measurable.

Proof. We assume that $p \neq q$. Suppose that $E$ is $\mu_{p}^{*}$-measurable and let $T$ be any subset of $X$. Suppose first that $T$ has a cover in $\mathcal{R}_{\sigma}$. Since both $T \cap E$ and $T \cap E^{c}$ have covers in $\mathcal{R}_{\sigma}$ and since $E$ is also $\mu^{*}$-measurable by Proposition 1 , we have from Lemma 10 (i) that

$$
\mu_{q}^{*}(T)=\mu^{*}(T)=\mu^{*}(T \cap E)+\mu^{*}\left(T \cap E^{c}\right)=\mu_{q}^{*}(T \cap E)+\mu_{q}^{*}\left(T \cap E^{c}\right) .
$$

Thus $E$ is $\mu_{q}^{*}$-measurable. Next suppose that $T$ has no cover in $\mathcal{R}_{\sigma}$ with $\mu_{q}^{*}(T)<\infty$. By Lemma 10 (iii) we have $\mu_{0}^{*}(T)<\infty$. By the assumption on 
$T$, at least one of the sets $T \cap E$ and $T \cap E^{c}$ has no cover in $\mathcal{R}_{\sigma}$. Suppose that both of these sets have no cover in $\mathcal{R}_{\sigma}$. Since $E$ is $\mu_{0}^{*}$-measurable by Propositions 1 and 2, we obtain from Lemma 10 (iii) that

$$
\begin{aligned}
\mu_{0}^{*}(T)+p & =\mu_{p}^{*}(T)=\mu_{p}^{*}(T \cap E)+\mu_{p}^{*}\left(T \cap E^{c}\right) \\
& =\mu_{0}^{*}(T \cap E)+\mu_{0}^{*}\left(T \cap E^{c}\right)+2 p=\mu_{0}^{*}(T)+2 p,
\end{aligned}
$$

so $p=0$. This is a contradiction. Therefore, we can assume that $T \cap E$ has a cover in $\mathcal{R}_{\sigma}$ and $T \cap E^{c}$ has no cover in $\mathcal{R}_{\sigma}$. Consequently, we have from parts (i) and (iii) of Lemma 10 that

$\mu_{q}^{*}(T)=\mu_{0}^{*}(T)+q=\mu_{0}^{*}(T \cap E)+\mu_{0}^{*}\left(T \cap E^{c}\right)+q=\mu_{q}^{*}(T \cap E)+\mu_{q}^{*}\left(T \cap E^{c}\right)$.

Thus $E$ is $\mu_{q}^{*}$-measurable. Interchanging $p$ and $q$ in the preceding result we establish the proposition.

Now we formulate the main result of this paper.

Theorem 2. $\mathcal{M}_{p}=\mathcal{M}_{1} \subset \mathcal{M}_{0}=\mathcal{M}_{\infty}=\mathcal{M}$ for all $p \in(0, \infty)$.

Proof. By Proposition 3 we have that $\mathcal{M}_{p}=\mathcal{M}_{1}$ for all positive real numbers p. We infer from Propositions 1 and 2 that $\mathcal{M}_{1} \subset \mathcal{M}=\mathcal{M}_{0}$. As we noted earlier, we have that $\mathcal{M}=\mathcal{M}_{\infty}$.

We need not have $\mathcal{M}_{1}=\mathcal{M}$ (see Example 5 or 6 below).

Example 5. Let $X=\{1,2\}, \mathcal{R}=\{\emptyset\}$, and $\mathcal{A}=\{\emptyset, X\}$. Plainly $\mathcal{R}$ is a $\sigma$-ring in $X$ with $X \notin \mathcal{R}$, and $\mathcal{A}$ is the $\sigma$-algebra generated by $\mathcal{R}$. Define $\mu(\emptyset)=0$. Then $\mu$ is a countably additive measure on $\mathcal{R}$. We have at once that $\mu^{*}(\emptyset)=0$ and $\mu^{*}(E)=\infty$ if $E$ is a nonempty subset of $X$, so $\mu^{*}$ is also a countably additive measure on $\mathcal{P}(X)=\{\emptyset,\{1\},\{2\}, X\}$. Thus $\mathcal{M}=\mathcal{P}(X)$. Since $\mu_{0}(\emptyset)=\mu_{0}(X)=0$, we obtain that $\mu_{0}^{*}(E)=0$ for all $E \subset X$. By Lemmas 5 and 10 we have that, for all $p \in(0, \infty], \mu_{p}(\emptyset)=0$ and $\mu_{p}(X)=p$, so $\mu_{p}^{*}(\emptyset)=0$ and $\mu_{p}^{*}(E)=p$ if $E=\{1\},\{2\}$ or $X$. Notice that $\mu^{*}=\mu_{\infty}^{*}$. We assert that $\mathcal{M}_{p}=\mathcal{A}$ for all $p \in(0, \infty)$. To prove the assertion, suppose that $p$ is any positive real number. Since $p=\mu_{p}^{*}(X)<\mu_{p}^{*}(\{1\})+\mu_{p}^{*}(\{2\})=2 p$, both $\{1\}$ and $\{2\}$ are not $\mu_{p}^{*}$-measurable and hence the assertion is established. Thus $\mathcal{M}_{1} \neq \mathcal{M}$.

In the following examples, let $X$ denote any uncountable set, let $\mathcal{R}$ denote the ring of all finite subsets of $X$, and let $\mathcal{A}$ and $\mathcal{B}$ denote, respectively, the algebra and the $\sigma$-algebra generated by $\mathcal{R}$ as in Example 2. For any measure $\mu$ on $\mathcal{R}$, the measure $\mu_{p}(0 \leq p \leq \infty)$ on $\mathcal{A}$ induced by $\mu$ and parameter $p$ is countably additive, since $\mu$ is countably additive. 
Example 6. Define $\mu(E)=0$ for all $E$ in $\mathcal{R}$. Plainly $\mu$ is a countably additive measure on $\mathcal{R}$. Since $\bar{\mu}(E)=0$ for all $E$ in $\mathcal{R}_{\sigma}$, we obtain that $\mu^{*}(E)=0$ or $\infty$ according as $E$ is countable or not. Then $\mu^{*}$ is also a countably additive measure on $\mathcal{P}(X)$ so that $\mathcal{M}=\mathcal{P}(X)$. We next compute $\mu_{0}^{*}$. Since $\mu_{0}(E)=0$ for all $A$ in $\mathcal{A}$, we obtain that $\bar{\mu}_{0}(E)=0$ for all $E$ in $\mathcal{A}_{\sigma}=\mathcal{R}_{\sigma} \cup \mathcal{R}^{c}$ (see Example 2), and hence $\mu_{0}^{*}(E)=0$ for all $E \subset X$. By Lemma 10 (iii) we have that $\mu_{1}^{*}(E)=0$ or 1 according as $E$ is countable or not. Assert that $\mathcal{M}_{1}=\mathcal{B}$. Since $\mathcal{B}=\mathcal{R}_{\sigma} \cup\left(\mathcal{R}_{\sigma}\right)^{c}$ by Example 2, we obtain $\mathcal{B} \subset \mathcal{M}_{1}$. To prove the opposite inclusion, suppose that $A$ is any subset of $X$ such that both $A$ and $A^{c}$ are uncountable. Then $1=\mu_{1}^{*}(X)<\mu_{1}^{*}(A)+\mu_{1}^{*}\left(A^{c}\right)=2$ so $A$ is not $\mu_{1}^{*}$-measurable. Thus the assertion holds. Consequently, $\mathcal{M}_{1} \neq \mathcal{M}$.

Example 7. Define $\mu$ on $\mathcal{R}$ by $\mu(E)=\operatorname{card} E$ for all $E$ in $\mathcal{R}$. Then $\mu$ is a countably additive measure on $\mathcal{R}$. We obtain that $\bar{\mu}(E)=\operatorname{card} E$ if $E$ is finite and $\bar{\mu}(E)=\infty$ if $E$ is countably infinite, so that $\mu^{*}(E)=\operatorname{card} E$ if $E$ is finite and $\mu^{*}(E)=\infty$ if $E$ is infinite. Then $\mu^{*}$ is a countably additive measure on $\mathcal{P}(X)$ so $\mathcal{M}=\mathcal{P}(X)$. On the other hand, we have that $\mu_{0}(E)=\operatorname{card} E$ if $E$ is finite and $\mu_{0}(E)=\infty$ if $E$ is cofinite, and that $\bar{\mu}_{0}(E)=$ card $E$ if $E$ is finite and $\bar{\mu}_{0}(E)=\infty$ if $E$ is countably infinite or cofinite. Consequently, $\mu_{0}^{*}=\mu^{*}$. By part (iv) of Lemma 10 we obtain that for all $p \in[0, \infty], \mu_{p}^{*}=\mu^{*}$ so $\mathcal{M}_{p}=\mathcal{M}$.

Example 8. Define $\mu$ on $\mathcal{R}$ by $\mu(\emptyset)=0$ and $\mu(E)=\infty$ if $E \in \mathcal{R}$ and $E \neq \emptyset$. Then $\mu$ is a countably additive measure on $\mathcal{R}$. It follows easily that $\mu^{*}(\emptyset)=0$ and $\mu^{*}(E)=\infty$ if $E \neq \emptyset$, and that $\mu^{*}$ is a countably additive measure on $\mathcal{P}(X)$, so $\mathcal{M}=\mathcal{P}(X)$. As in Example 7, we obtain that for all $p \in[0, \infty]$, $\mu_{p}^{*}=\mu^{*}$ so $\mathcal{M}_{p}=\mathcal{M}$.

Acknowledgment. The authors wish to thank the referee for valuable remarks and suggestions.

\section{References}

[1] K. P. S. Bhaskara Rao and M. Bhaskara Rao, Theory of Charges, Academic Press, London,1983.

[2] P. Billingsley, Probability and Measure, 2nd ed., Wiley, New York, 1986.

[3] J. L. Doob, Measure Theory, Springer-Verlag, New York,1991.

[4] M. M. Rao, Measure Theory and Integration, Wiley, New York, 1987.

[5] H. L. Royden, Real Analysis, 3rd ed., Macmillan, New York, 1988. 